Claremont Colleges

Scholarship@ Claremont

All HMC Faculty Publications and Research

HMC Faculty Scholarship

$1-1-2003$

\title{
Breakdown of the Slowly Varying Amplitude Approximation: Generation of Backward Traveling Second Harmonic Light
}

\author{
J.Z. Sanborn '01 \\ Harvey Mudd College \\ C. Hellings '02 \\ Harvey Mudd College \\ Thomas D. Donnelly \\ Harvey Mudd College
}

\section{Recommended Citation}

J. Z. Sanborn, C. Hellings, T. D. Donnelly, "Breakdown of the slowly varying amplitude approximation: generation of backward traveling second harmonic light," J. Opt. Soc. A. B, 20, 152-157 (2003). doi: 10.1364/JOSAB.20.000152

This Article is brought to you for free and open access by the HMC Faculty Scholarship at Scholarship @ Claremont. It has been accepted for inclusion in All HMC Faculty Publications and Research by an authorized administrator of Scholarship @ Claremont. For more information, please contact scholarship@cuc.claremont.edu. 


\title{
Breakdown of the slowly-varying-amplitude approximation: generation of backward-traveling, second-harmonic light
}

\author{
J. Z. Sanborn, C. Hellings, and T. D. Donnelly \\ Department of Physics, Harvey Mudd College, Claremont, California 91711
}

Received December 20, 2001; revised manuscript received August 22, 2002

\begin{abstract}
By numerically solving the nonlinear field equations, we simulate second-harmonic generation by laser pulses within a nonlinear medium without making the usual slowly-varying-amplitude approximation, an approximation which may fail when laser pulses of moderate intensity or ultrashort duration are used to drive a nonlinear process. Under these conditions we show that a backward-traveling, second-harmonic wave is created, and that the magnitude of this wave is indicative of the breakdown of the slowly-varying-amplitude approximation. Conditions necessary for experimental detection of this wave are discussed. (C) 2003 Optical Society of America

OCIS codes: $190.0190,190.4160,350.5500$.
\end{abstract}

The slowly-varying-amplitude approximation (SVAA) is regularly used to simplify the analysis of light's interaction with nonlinear optical media. ${ }^{1,2}$ The SVAA allows one to assume that the amplitude of the light incident on the optical medium changes negligibly over distance scales roughly equal to the light's wavelength or, equivalently, over time scales roughly equal to the light's period. This simplifies the analysis greatly because it rids the theoretical treatment of all second-order and higher time and distance derivatives when compared with the much larger first-order terms. This approximation is invoked nearly universally to describe nonlinear optical phenomena such as harmonic generation, stimulated light scattering (e.g., Raman and Brillouin scattering), fourwave mixing, self-focusing, and nonlinearities in waveguides. $^{1-3}$ It is well known that making the SVAA is equivalent to ignoring a backward-traveling wave propagating at the fundamental frequency.

The SVAA begins to fail when the incident light is moderately intense or when, in the case of ultrashort laser pulses, the pulse envelope changes significantly over the course of a single optical cycle. The inability to use the SVAA significantly complicates the analysis of many nonlinear interactions, rendering it impossible to form an analytic solution to the nonlinear wave equation. However, many studies have investigated implications of the SVAA breaking down, ${ }^{4-9}$ and in the purely time-varying domain (where it is known as the rotating-wave approximation) analytic corrections to it are known. ${ }^{10,11}$ Casperson ${ }^{6-8}$ has shown that instabilities are predicted in steady-state laser amplifiers if the SVAA is not used. In a study of how femtosecond pulses interact with a twolevel atom Hughes $^{9}$ finds the production of highfrequency spectral components on the propagating pulse which are not predicted when the SVAA is made. Brabec and Krausz ${ }^{5}$ show that, in practice, many nonlinear phenomena can be accurately described using the SVAA even if the driving pulse is a single cycle of radiation. How- ever their analysis does not apply to the backwardtraveling radiation discussed here.

Here we study numeric solutions to the nonlinear wave equation without making the SVAA. We focus on a particular second-order effect, the generation of a backwardtraveling, second-harmonic wave, to quantify the physical importance of the second-order terms ignored by the SVAA. Analytical solutions that demonstrate the existence of the backward-traveling, second-harmonic wave have been found but require a set of conditions that are hard to realize experimentally. Specifically the current studies of this phenomenon assume phase matching in both forward and reverse directions, as well as that no attenuation of the pump field occurs within the optical medium. ${ }^{11}$ Although these assumptions may indeed be applicable to special classes of media, they are invalid in the general case. In our numerical approach to this problem the above assumptions become unnecessary. Therefore the following analysis develops a more feasible method of experimentally and quantitatively studying the backward-traveling, second-harmonic waves predicted by the numerical approach.

In the following analysis we find steady-state solutions to the nonlinear wave equation that assume an incident plane wave. The results we obtain are for steady-state conditions and thus will apply precisely to continuous wave lasers and hold approximately for laser pulses of adequately long duration $\tau \gg l / v$, where $\tau$ is the pulse width, $v$ is the group velocity of the fundamental pulse, and $l$ is the characteristic interaction length of the medium.

The field equations describing second-harmonic generation (SHG) in steady state can be derived from Maxwell's equations when a nonlinear polarizability $\widetilde{P}^{\mathrm{NL}}$ of the medium is assumed to be present. ${ }^{3}$ We start with

$$
\nabla \times \nabla \times \widetilde{E}+\frac{n^{2}}{c^{2}} \frac{\partial^{2} \widetilde{E}}{\partial t^{2}}=\frac{-4 \pi}{c^{2}} \frac{\partial^{2} \widetilde{P}^{\mathrm{NL}}}{\partial t^{2}} .
$$


We assume a steady-state solution for the field, varying in time as

$$
\widetilde{E}_{1}(z, t)=E_{1}(z) \exp \left(-i \omega_{1} t\right)+\text { c.c. }
$$

and we factor out the rapidly varying part of the field according to

$$
E_{1}(z)=A_{1}(z) \exp \left(i k_{1} z\right) .
$$

The second-order polarizations are given by

$$
\begin{aligned}
& P_{1}(z)=4 d_{\mathrm{eff}} E_{1}^{*}(z) E_{2}(z), \\
& P_{2}(z)=2 d_{\mathrm{eff}} E_{1}^{2}(z) .
\end{aligned}
$$

These polarizations provide the field with a source of new components, particularly the second-harmonic wave that is the focus of this discussion. Using the above definitions, we can express the wave Eq. (1) as the two, coupled, nonlinear differential equations

$$
\begin{aligned}
& \frac{\mathrm{d}^{2} A_{1}}{\mathrm{~d} z^{2}}+2 i k_{1} \frac{\mathrm{d} A_{1}}{\mathrm{~d} z}=-\frac{16 d_{\mathrm{eff}} \pi \omega_{1}^{2}}{c^{2}} A_{1}^{*} A_{2} \exp (-i \Delta k z), \\
& \frac{\mathrm{d}^{2} A_{2}}{\mathrm{~d} z^{2}}+2 i k_{2} \frac{\mathrm{d} A_{2}}{\mathrm{~d} z}=-\frac{8 d_{\mathrm{eff}} \pi \omega_{2}^{2}}{c^{2}} A_{1}^{2} \exp (i \Delta k z),
\end{aligned}
$$

where $\Delta k$ is the phase mismatch $\left(2 k_{1}-k_{2}\right)$ between the forward-traveling fundamental and second-harmonic waves and the indices $i=1,2$ correspond to the fundamental and second-harmonic waves.

We introduce a unitless distance parameter $\xi=z / l$ for which the characteristic interaction length $l$ is given $b^{3}$

$$
l=\frac{1}{8 \pi \omega_{1} d_{\mathrm{eff}}}\left(\frac{n_{1}{ }^{2} n_{2} c^{3}}{2 \pi I}\right)^{1 / 2},
$$

where $n_{i}$ is the index of refraction for the $i$ th harmonic frequency component, $\omega_{1}$ is the field's fundamental frequency, $d_{\text {eff }}$ is the medium's effective second-order susceptibility, and $I$ is total intensity (we know from the Manley-Rowe relations that $I=I_{1}+I_{2}$ is spatially invariant in second-harmonic generation).

In a linear medium the complex amplitude of a plane wave is constant. This is not the case in a nonlinear medium, thus it is useful to represent the complex, slowlyvarying wave amplitude as $A_{i}=\left(2 \pi I / n_{i} c\right)^{1 / 2} u_{i} \exp \left(i \phi_{i}\right)$. Using this substitution in Eqs. (6) and (7) and separating real and imaginary parts, we obtain

$$
\begin{aligned}
-a_{1} u_{1}^{\prime \prime}+a_{1} u_{1}\left(\phi_{1}^{\prime}\right)^{2} & +u_{1} \phi_{1}^{\prime} \\
& =u_{1} u_{2} \cos \left(\Delta s \xi+2 \phi_{1}-\phi_{2}\right), \\
2 a_{1} u_{1}^{\prime} \phi_{1}^{\prime}+a_{1} u_{1} \phi_{1}^{\prime \prime} & +u_{1}^{\prime} \\
& =u_{1} u_{2} \sin \left(\Delta s \xi+2 \phi_{1}-\phi_{2}\right), \\
-a_{2} u_{2}^{\prime \prime}+a_{2} u_{2}\left(\phi_{2}^{\prime}\right)^{2} & +u_{2} \phi_{2}^{\prime} \\
& =u_{1}{ }^{2} \cos \left(\Delta s \xi+2 \phi_{1}-\phi_{2}\right), \\
2 a_{2} u_{2}^{\prime} \phi_{2}^{\prime}+a_{2} u_{2} \phi_{2}^{\prime \prime}+ & u_{2}^{\prime} \\
& =-u_{1}^{2} \sin \left(\Delta s \xi+2 \phi_{1}-\phi_{2}\right),
\end{aligned}
$$

where $a_{i}=1 / 2 k_{i} l$ and the primes indicate derivatives with respect to $\xi$. These four equations are solved numerically using a Runge-Kutta ${ }^{4,5}$ algorithm assuming phase matching only in the forward direction $\Delta s=\Delta k l$ $=0$.

It can be seen from Eq. (8) that the characteristic interaction length $l$ depends both on the intensity of the laser pulse and the effective second-order susceptibility $d_{\text {eff }}$. As either the intensity or $d_{\text {eff }}$ is increased the interaction length decreases, thereby decreasing the distance in which the fundamental and second-harmonic fields exchange energy. Consequently if the interaction length gets to be roughly the same size as the wavelength of the field, the SVAA $\left[\partial^{2} A / \partial z^{2} \ll k \partial(A / \partial z)\right]$ becomes less valid and the second-order terms usually neglected through the SVAA may become important. An intense titanium:sapphire pulse $\left(\omega_{1}=2.36 \times 10^{15} \mathrm{~Hz}, I=1 \times 10^{12} \mathrm{~W} / \mathrm{cm}^{2}\right)$ traveling through a lithium niobate crystal $\left(d_{\text {eff }} \approx 5\right.$ $\times 10^{-8} \mathrm{esu}, n_{1} \approx n_{2} \approx 2$ ) will have an interaction length $l \approx 6 \mu \mathrm{m} ;$ if the nonlinear crystal is $\mathrm{KDP} \quad\left(d_{\mathrm{eff}} \approx 1\right.$ $\times 10^{-9} \mathrm{esu}, n_{1} \approx n_{2} \approx 1.5$ ) the interaction length is longer, $l \approx 200 \mu \mathrm{m}$. Therefore for the lithium niobate crystal the "steady-state" approximation $(\tau \gg l / v)$ will hold for $\tau \gg 40 \mathrm{fs}$ - a reasonable assumption for most laser pulses capable of reaching the required intensity.

Notice that the parameters $a_{i}$ have the effect of controlling the relative strength of the second-order terms. Thus making the SVAA is equivalent to setting $a_{1}$ and $a_{2}$ equal to zero in Eqs. (9)-(12), and in this case the wellknown solutions for SHG are recovered and no backwardtraveling wave is predicted to exist.

To obtain the magnetic field from the numerical solutions of Eqs. (9)-(12) we use Faraday's law to obtain a relation between $\widetilde{E}$ and $\widetilde{B}$ as

$$
\frac{\partial \widetilde{E}(z, t)}{\partial z}=-\frac{1}{c} \frac{\partial \widetilde{B}(z, t)}{\partial t},
$$

which reduces to

$$
\frac{\partial \tilde{E}(z, t)}{\partial z}=\frac{i \omega \mu_{1}}{c} \tilde{H}(z, t),
$$

where $\widetilde{B}=\mu_{1} \widetilde{H} . \quad \widetilde{H}$ can be written in terms of a slowlyvarying field amplitude $M(z)$, much as we previously did in Eq. (3), to obtain

$$
\begin{aligned}
\tilde{H}_{i}(z, t) & =\left(\frac{\epsilon_{1}}{\mu_{1}}\right)^{1 / 2} H_{i}(z) \exp \left(-i \omega_{i} t\right) \\
& =\left(\frac{\epsilon_{1}}{\mu_{1}}\right)^{1 / 2} M_{i}(z) \exp \left(i k_{i} z\right) \exp \left(-i \omega_{i} t\right) .
\end{aligned}
$$

Eq. (14) then reduces to

$$
2 a A^{\prime}(\xi)+i A(\xi)=i M(\xi) .
$$

If we separate $A$ and $M$ into their real and imaginary parts in which $A=A_{r}+i A_{i}$ and $M=M_{r}+i M_{i}$, we obtain

$$
\begin{aligned}
2 a A_{r}^{\prime}(\xi)+2 a i A_{i}^{\prime}(\xi)+i A_{r}(\xi) & -A_{i}(\xi) \\
& =i M_{r}(\xi)-M_{i}(\xi) .
\end{aligned}
$$


We can separate this into its real and imaginary parts to obtain

$$
\begin{aligned}
& M_{r}(\xi)=2 a A_{i}^{\prime}(\xi)+A_{r}(\xi), \\
& M_{i}(\xi)=-2 a A_{r}^{\prime}(\xi)+A_{i}(\xi),
\end{aligned}
$$

providing us a formula for the magnetic field components in terms of the wave amplitudes $A$.

To elucidate the underlying physics of Eqs. (9)-(12) we separate the intensities of the fundamental and secondharmonic waves into forward- and backward-traveling components using methods similar to Casperson. ${ }^{6}$ This is possible if we specify both the magnetic and electric fields. The electric field can be decomposed into forward(+) and backward- (-) traveling components by noting that the electric field can be expressed as a superposition of forward- and backward-traveling waves in the form

$$
\begin{aligned}
\widetilde{E}(z, t) & =E^{+}(z) \exp (i k z-i \omega t)+E^{-}(z) \exp (-i k z-i \omega t) \\
& =\left[E^{+}(z)+E^{-}(z) \exp (-2 i k z)\right] \exp (i k z-i \omega t) .
\end{aligned}
$$

Similarly, the magnetic field can be represented as

$$
\begin{aligned}
\tilde{H}(z, t)= & \left(\frac{\epsilon_{1}}{\mu_{1}}\right)^{1 / 2}\left[H^{+}(z)+H^{-}(z) \exp (-2 i k z)\right] \\
& \times \exp (i k z-i \omega t) .
\end{aligned}
$$

By comparing Eqs. (20) and (2), we see that the field amplitudes $E(z)$ can be reduced to plus and minus components according to

$$
A(z)=E^{+}(z)+E^{-}(z) \exp (-2 i k z),
$$

and similarly

$$
M(z)=H^{+}(z)+H^{-}(z) \exp (-2 i k z) .
$$

In general the plus and minus components of the fields do not separately satisfy the nonlinear wave equation, only their sum does. However, we consider a region in the nonlinear medium small enough that the forward and backward components are exchanging a negligible amount of energy. The forward and backward components are then well defined as independent electromagnetic fields ${ }^{6}$ and we can use Ampere's and Faraday's laws to relate the electric and magnetic field components as

$$
\begin{aligned}
& E^{+}(z)=H^{+}(z), \\
& E^{-}(z)=-H^{-}(z),
\end{aligned}
$$

or

$$
\begin{aligned}
& A^{+}(z)=M^{+}(z), \\
& A^{-}(z)=-M^{-}(z) .
\end{aligned}
$$

Using the above relations we rewrite Eq. (22) as

$$
A(z)=A^{+}(z)+A^{-}(z) \exp (-2 i k z),
$$

and Eq. (23) as

$$
M(z)=A^{+}(z)-A^{-}(z) \exp (-2 i k z) .
$$

Combining Eqs. (28) and (29) we obtain the plus and minus components of the wave amplitudes for the electric field as

$$
\begin{aligned}
& A^{+}(z)=\frac{1}{2}[A(z)+M(z)], \\
& A^{-}(z)=\frac{1}{2}[A(z)-M(z)] \exp (2 i k z),
\end{aligned}
$$

and for the magnetic field as

$$
\begin{aligned}
& M^{+}(z)=\frac{1}{2}[M(z)+A(z)] \\
& M^{-}(z)=\frac{1}{2}[M(z)-A(z)] \exp (2 i k z) .
\end{aligned}
$$

From the Poynting vector we can now obtain the forwardand backward-traveling intensities ${ }^{8}$ as

$$
I^{ \pm}=\frac{c}{4 \pi}\left(A_{r}^{ \pm} M_{r}^{ \pm}+A_{i}^{ \pm} M_{i}^{ \pm}\right) .
$$

Now by using the plus and minus components of the electric and magnetic fields given by Eqs. (30) and (31), respectively, with Eq. (34) we obtain expressions for the instantaneous forward- and backward-traveling wave intensities in the form

$$
\begin{aligned}
& I^{+}(\xi)=\frac{c}{4 \pi}\left\{\frac{1}{4}\left[A_{r}(\xi)+M_{r}(\xi)\right]^{2}+\frac{1}{4}\left[A_{i}(\xi)+M_{i}(\xi)\right]^{2}\right\}, \\
& I^{-}(\xi)=\frac{c}{4 \pi}\left\{\frac{1}{4}\left[A_{r}(\xi)-M_{r}(\xi)\right]^{2}+\frac{1}{4}\left[A_{i}(\xi)-M_{i}(\xi)\right]^{2}\right\} .
\end{aligned}
$$

Since our numerical model will not be computing $B(z)$, we instead express (35) and (36) solely in terms of the wave amplitudes $A(z)$ for the electric field by using Eqs. (18) and (19). We then obtain two expressions for the forward- and backward-traveling wave intensities as

$$
\begin{aligned}
& I^{+}(\xi)=\frac{c}{4 \pi}\left\{\left[A_{r}(\xi)+a A_{i}^{\prime}(\xi)\right]^{2}+\left[A_{i}(\xi)-a A_{r}^{\prime}(\xi)\right]^{2}\right\}, \\
& I^{-}(\xi)=\frac{c}{4 \pi}\left\{\left[a A_{i}^{\prime}(\xi)\right]^{2}+\left[a A_{r}^{\prime}(\xi)\right]^{2}\right\} .
\end{aligned}
$$

Therefore the real and imaginary components of $A(z)$ are all that is required to compute the intensity of the forward and backward traveling waves. For instance the intensity of the backward-traveling wave is given simply by the spatial derivatives of the electric field at the point of interest, as expected since for a linear medium $A_{i}^{\prime}=0$. As noted previously, setting $a_{i}$ to zero is equivalent to invoking the SVAA, and Eq. (38) shows that in this case the backward-traveling wave vanishes. Conversely the magnitude of the backward-traveling wave is indicative of how severely the SVAA breaks down.

Knowing the form of the intensities of the forward- and backward-traveling waves, we now analyze the physically imposed boundary conditions that are required for the numerical analysis.

To solve Eqs. (9)-(12), boundary conditions must be established. We assume a plane wave incident on a non- 
linear medium of length $L$, as shown in Fig. 1. An antireflective coating is assumed on the crystal's output so that no forward-traveling waves are reflected from the output face of the crystal; therefore $I_{1}^{-}(L)=0$ and $I_{2}^{-}(L)=0$. The forward intensities of the fundamental and second harmonic are normalized to unity at the output, constraining another boundary value $I_{2}^{+}(L)$ $=\left[1-I_{1}^{+}(L)^{2}\right]^{1 / 2}$. At the input face of the crystal $z=0$, we impose the physical constraint that there be no forward-traveling second harmonic present; that is, $I_{2}^{+}(0)=0$. This is because at the input there is not yet any medium in which to transfer energy from the fundamental field to the second harmonic. We further require that the first derivative of the phases be set to zero at the output face since, from a physical standpoint, the phases must go smoothly to constants when exiting the material; therefore $\phi_{1}^{\prime}(L)=0, \phi_{2}^{\prime}(L)=0$. These six boundary conditions are the only constraints we may impose on the system, leaving two more to fulfill the eight required for a second-order, four-variable, boundary value problem. We can simplify our analysis by noting that Eqs. (9)-(12) depend initially only on the difference of the fundamental and second-harmonic phase, which allows a single boundary condition to represent their overall initial difference $\theta=2 \phi_{1}-\phi_{2}$.

Since we have more constraints at the output of the crystal than at the input, we will simplify the numerical analysis by solving Eqs. (9)-(12) backwards, from the output to the input face. The remaining two boundary conditions at the output face must be adjusted recursively until the constraint that no forward-traveling, secondharmonic wave is present at the input face is satisfied. The boundary conditions to be iteratively adjusted are the values of $I_{1}^{+}(L)$ and $\theta$ at the output face.

We use a numerical algorithm to find the values of $I_{1}^{+}(L)$ and $\theta$ at the output that satisfy the boundary condition at the input. In the first iteration, it takes the parameter $a_{1}$ and the user's guesses for the initial values of $I_{1}^{+}(L)$ and $\theta$ and uses them to solve Eqs. (9)-(12) backwards from the output face. It then finds the location of

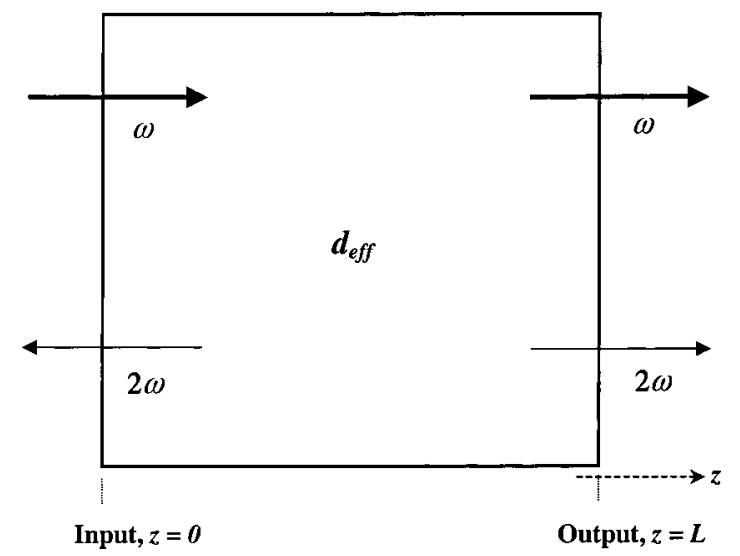

Fig. 1. Box represents the nonlinear medium and the fundamental wave is incident from the left. As discussed in the text, the eight boundary conditions applied for the numeric analysis are (1) $I_{1}^{-}(L)=0,(2) I_{2}^{-}(L)=0,(3)\left[I_{2}^{+}(L)\right]^{2}=1-\left[I_{1}^{+}(L)\right]^{2},(4)$ $I_{2}^{+}(0)=0,(5) \phi_{1}^{\prime}(L)=0$, (6) $\phi_{2}^{\prime}(L)=0$, (7) $\theta=2 \phi_{1}-\phi_{2}=$ ?, and (8) $I_{1}^{+}(L)=$ ? A question mark denotes a boundary condition that is unknown and must be found self-consistently. the minimum intensity of the forward-traveling second harmonic within its first solution, which is an estimate for the location of the crystal's input face (i.e., where $L$ $=0$ ). This length is the region that will be integrated over to find subsequent solutions. The algorithm will now recursively alter the value of $\theta$ to find the local minimum value of $I_{2}^{+}(0)$ with the given user-defined parameters $I_{1}^{+}(L)$ and $a_{1}$. It does this by stepping the value of $\theta$ in the direction that decreases the value of $I_{2}^{+}(0)$, halving the step size when near the local minimum to find the local minimum within a given tolerance. This tolerance is set at $0.001 \%$, meaning that a $0.001 \%$ change in the value of $I_{2}^{+}(0)$ from one iteration to the next will satisfy the tolerance, and the local minimum will have been found to the desired accuracy. When the algorithm determines the location of the minimum of $I_{2}^{+}(0)$ in phase space, it outputs the value of $I_{2}^{+}(0)$, the phase angle $\theta$ at which the minimum occurs, and the length $L$ of the crystal. At this point the user decides if the passed-in value of $I_{2}^{+}(L)$ must be changed to achieve either a lower value of $I_{2}^{+}(0)$ or a different crystal length $L$ (for example one that conforms with an experiment).

With the boundary conditions found using the numerical algorithm as described above we can study the generation of the backward-traveling, second-harmonic wave. Figure 2 shows the forward- and backward-traveling waves calculated for the fundamental and secondharmonic waves when $a=0.01$. Figure 2(a) shows the usual exchange of energy between the forward-traveling fundamental and second-harmonic waves which occurs over the first few interaction lengths. Figure 2(b) demonstrates the growth of backward-traveling fundamental and second-harmonic waves. The higher-order oscillations result from standing waves within the nonlinear crystal.

The low efficiency of backward SHG shown in Fig. 2(b) is a result of the mismatch in the phases of the fundamental and second-harmonic fields in the backward direction. It is possible to match the phases in the forward direction both experimentally and numerically (by setting $\Delta s$ $=0$ ), but the total phase mismatch in the backward direction is given by $\left[\left(2 / a_{1}\right)+\left(1 / a_{2}\right)\right]$, which shows the impossibility of phase matching. However, it has been shown that by using periodically poled crystals it is possible to quasi-match the phases in the backward process. ${ }^{12-14}$ By quasi-matching the backward process more growth of the backward-traveling wave is allowed, thereby resulting in a more efficient process ( $~ 8 \%$ conversion efficiency by poling lithium niobate with a $0.19-\mu \mathrm{m}$ period). Current analytical approximations for backward $\mathrm{SHG}^{12}$ depend on quasi-matching of phases (and therefore the use of periodically-poled crystals) and negligible depletion of the pump field, two assumptions not required by the numerical analysis presented in this paper.

Figure 3 shows the backward SHG efficiency (normalized to the input fundamental wave) as a function of the parameter $a_{1}$. This shows an increase in the secondharmonic efficiency as $a_{1}$ increases. Calculations are performed only up to values of $a_{1} \approx 0.02$ because materials will certainly sustain damage at corresponding intensities above this value. For example we know from Eq. (8) and the definition of $a_{1}$ that 

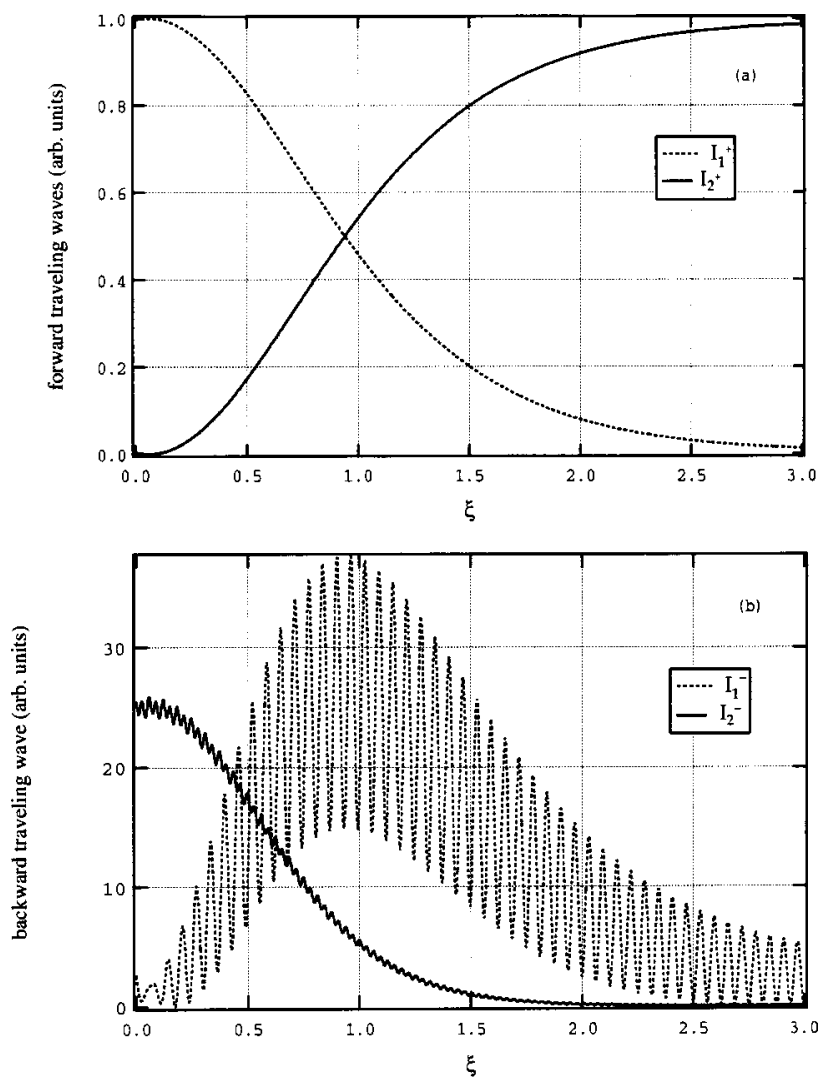

Fig. 2. (a) Forward- and (b) backward-traveling waves plotted as a function of $\xi$ for $a_{1}=0.01$. The growth of a backwardtraveling, second-harmonic wave is evident in (b) as is the existence of a backward-traveling wave at the fundamental frequency. If the nonlinear medium is taken to be lithium niobate $\left(d_{\text {eff }} \approx 5 \times 10^{-8} \mathrm{esu}\right)$ then for typical laser parameters $\left(\omega_{1}\right.$ $\left.=2.36 \times 10^{15} \mathrm{~Hz}, I=10^{12} \mathrm{~W} / \mathrm{cm}^{2}\right)$ the unitless length $\xi=3$ corresponds to a crystal length of $18 \mu \mathrm{m}$. Note that if the the SVAA approximation is invoked, no backward-traveling waves exist.

$$
I=\frac{n_{1}{ }^{4} n_{2} c}{32 \pi^{3} d_{\mathrm{eff}}^{2}} a_{1}{ }^{2},
$$

and therefore in lithium niobate $a_{1}=0.016$ corresponds to an intensity of approximately $1 \times 10^{13} \mathrm{~W} / \mathrm{cm}^{2}$. On the other end of the scale, for lithium niobate $a_{1}$ $=0.0005$ corresponds to an intensity of approximately $1 \times 10^{10} \mathrm{~W} / \mathrm{cm}^{2}$.

Figure 3 suggests the feasibility of experimentally verifying the breakdown of the SVAA: The growth of the backward-traveling, second-harmonic wave in a nonlinear medium can be monitored as a function of the input laser intensity and quantitatively compared to the predicted efficiencies.

Such an experiment to test the breakdown of the SVAA and the numeric modeling presented here would use lithium niobate as the nonlinear medium. Lithium niobate has a large $d_{\text {eff }}$ compared with KDP or BBO, meaning that to reach a nominal $a$ value, and therefore a desired efficiency in generating a backward-traveling, second-harmonic wave, a lower laser intensity can be used for lithium niobate than for KDP or BBO. For example to reach an efficiency of $10^{-5}$ for the backward- traveling, second-harmonic wave, a value of approximately $a=0.005$ is necessary (see Fig. 3 ). This implies a laser intensity of $10^{12} \mathrm{~W} / \mathrm{cm}^{2}$ for lithium niobate and $6 \times 10^{14} \mathrm{~W} / \mathrm{cm}^{2}$ for $\mathrm{KDP}$, which is above the damage threshold. Also any possibility of self-focusing should be avoided so that knowledge of the pump laser's intensity can be ensured. This consideration, together with consideration of damage thresholds, further constrains the choice of nonlinear media. To minimize pump power (to avoid self-focusing) a material is necessary that responds relatively strongly in second order to lower intensities, suggesting lithium niobate with a large $d_{\text {eff }}$ as the material of choice. (Note that while the critical power for selffocusing depends inversely on $d_{\text {eff }}$, reaching a particular choice of $a$ value depends quadratically on $d_{\text {eff }}$ and therefore there is an advantage to choosing materials of large $d_{\text {eff }}$.) Finally a larger $d_{\text {eff }}$ implies a shorter interaction length $l$ and thus relatively short "steady-state" time $\tau$. For conditions listed above $\left(\omega_{1}=2.36 \times 10^{15} \mathrm{~Hz}, I=1\right.$ $\times 10^{12} \mathrm{~W} / \mathrm{cm}^{2}$ ) lithium niobate has an interaction length of $6 \mu \mathrm{m}$ and KDP has an interaction length of $200 \mu \mathrm{m}$; this implies $\tau_{l-n}=40 \mathrm{fs}$ while $\tau_{\mathrm{KDP}}=1 \mathrm{ps}$. Using lithium niobate therefore means that our analysis would apply if typical titanium:sapphire laser pulses (pulse duration $\gg 40 \mathrm{fs}$ ) were used to pump the nonlinear interaction.

Thus a simple experiment can be performed using a titanium:sapphire pump laser and a lithium niobate crystal. The lithium niobate crystal should be antireflection coated so that the boundary conditions listed in Fig. 1 are met. The pump laser can be focused onto a crystal which is misaligned from normal incidence (this avoids collecting spurious back reflections along with the desired backward-generated, second-harmonic light), thus generating backward-going, second-harmonic light which can be collected with a dichroic beam splitter. The signal from the backward-traveling light can be monitored using a photomultiplier tube and compared to the intensity of the pump laser. In this way the predictions shown in Fig. (3) can be tested.

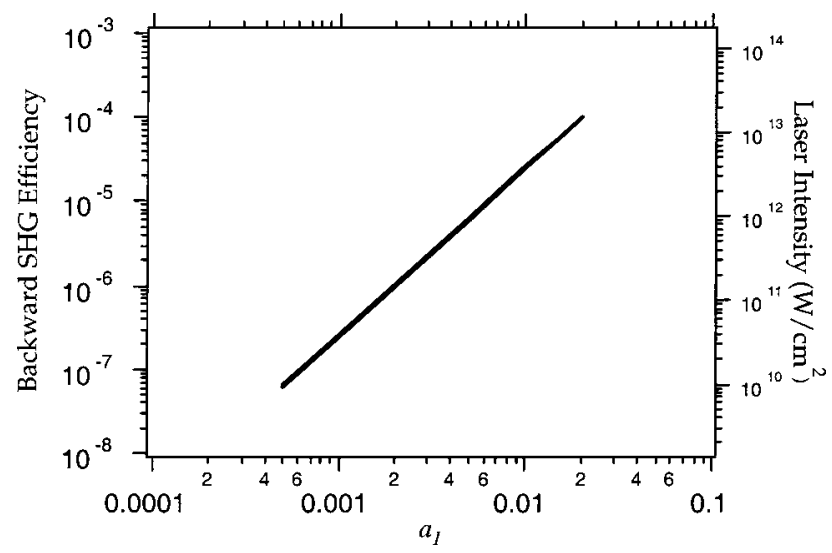

Fig. 3. Backward SHG efficiency versus parameter $a_{1}$. At pump-laser intensities above $10^{10} \mathrm{~W} / \mathrm{cm}^{2}$ the backward second harmonic can be detected. The calculation is truncated for laser intensities greater than $10^{13} \mathrm{~W} / \mathrm{cm}^{2}$ since damage of the nonlinear medium would be expected for higher intensities. The intensity axis is calculated assuming lithium niobate as the nonlinear medium. 
In summary, we have shown that in a moderate intensity regime the second-order terms in the nonlinear wave equation must be included to model the full response of the nonlinear system. The emergence of a backwardtraveling, second-harmonic wave is analyzed. Its generation efficiency is calculated and related to the breakdown of the SVAA. An experiment to measure the backward-traveling, second-harmonic wave is outlined.

\section{ACKNOWLEDGMENTS}

We thank Lee Casperson and Andrew Bernoff for helpful conversations. Acknowledgment is made to the Donors of The Petroleum Research Fund, administered by the American Chemical Society, for partial support of this research, and for support under an award from the Research Corporation.

T. D. Donnelly may be reached by e-mail at tom_donnelly@hmc.edu.

\section{REFERENCES AND NOTES}

1. J. A. Armstrong, N. Bloembergen, J. Ducuing, and P. S. Pershan, "Interactions between light waves in a nonlinear dielectric," Phys. Rev. 127, 1918-1939 (1962).

2. Y. R. Shen, The Principles of Nonlinear Optics (Wiley, New York, 1984)

3. R. W. Boyd, Nonlinear Optics (Academic, Boston, Mass., 1992).
4. S. E. Harris, "Proposed backward wave oscillation in the infrared," Appl. Phys. Lett. 9, 114-116 (1966).

5. T. Brabec and F. Krausz, "Nonlinear optical pulse propagation in the single-cycle regime," Phys. Rev. Lett. 78, 32823285 (1997).

6. L. W. Casperson, "Field-equation approximations and amplification in high gain lasers: numeric results," Phys. Rev. A 44, 3291-3304 (1991).

7. L. W. Casperson, "Field-equation approximations and amplification in high gain lasers: analytic results," Phys. Rev. A 44, 3305-3316 (1991).

8. L. W. Casperson, "Field-equation approximation and the dynamics of high-gain lasers," Phys. Rev. A 43, 5057-5067 (1991).

9. S. Hughes, "Breakdown of the area theorem: carrier-wave rabi flopping of femtosecond optical pulses," Phys. Rev. Lett. 81, 3363-3366 (1998).

10. F. Bloch and A. J. Siegert, "Magnetic resonance for nonrotating fields," Phys. Rev. 57, 522-527 (1940).

11. For a discussion of the rotating-wave approximation in the context of optical phenomena see L. Allen and J. H. Eberly, Optical Resonance and Two-Level Atoms (New York, Dover, 1987), Chap. 2.

12. Y. J. Ding, J. U. Kang, and J. B. Khurgin, "Theory of backward second-harmonic and third-harmonic generation using laser pulses in quasi-phase-matched second-order nonlinear medium,” IEEE J. Quantum Electron. 34, 966-974 (1998).

13. X. Gu, R. Y. Korotkov, Y. J. Ding, J. U. Kang, and J. B. Khurgin, "Backward second-harmonic generation in periodically poled lithium niobate," J. Opt. Soc. Am. B 15, 1561-1566 (1998).

14. X. H. Gu, M. Makarov, Y. J. Ding, J. B. Khurgin, and W. P. Risk, "Backward second-harmonic and third-harmonic generation in a periodically poled potassium titanyl phosphate waveguide," Opt. Lett. 24, 127-129 (1999). 